\title{
The uncertain pathway to new therapeutics for SLE
}

\author{
Peter E Lipsky
}

Two abstracts presented at the latest American College of Rheumatology/Association of Rheumatology Health Professionals Scientific Meeting reported failures to achieve primary outcomes in patients with systemic lupus erythematosus (SLE) treated with rituximab or abatacept (Merrill JT et al. [2008] late breaking abstracts L12 and L15). Together with the lack of success of belimumab (Furie R et al. [2008] Arthritis Res Ther 10: R109), these results have raised important questions about the prospects for safe and effective new therapies for SLE, the design of clinical trials in SLE, the appropriate selection of patients for these trials, the pharmacodynamics of these agents in humans, and the immunopathogenesis of SLE itself.

Since the development of the LE cell test and the discovery of anti-nuclear antibodies over 50 years ago, it has been widely accepted that the pathophysiology of SLE is mediated by immune cell activity. If this is true, how can it be that therapies directed at either B cells (e.g. rituximab and belimumab) or T cells (e.g. abatacept) have failed to have a clinical impact in SLE? The preclinical and clinical information supporting a role for antibodies, B cells and T cells in SLE is quite convincing; therefore, it is unlikely that the trials have revealed a flaw in the scientific rationale for testing these agents in SLE. In fact, broader immunosuppressive agents, such as cyclophosphamide, azathioprine and mycophenolate mofetil, are effective, although none has been approved for the treatment of SLE in the US. So, what is the problem, and what can we learn that will stimulate development of new therapeutics for SLE, as broad spectrum immunosuppressive agents are associated with numerous undesirable side effects?

The agents tested in SLE clinical trials are all quite specific, focusing on a particular component of the immune system. Therefore, it is

\section{The pathway \\ to new agents \\ for SLE is likely \\ to be similar \\ [to that of RA], \\ with a number \\ of fits and \\ starts until a \\ successful \\ agent defines \\ the nature \\ of the \\ approach that \\ will succeed.}

PE Lipsky is

the Editor-in-

Chief of Nature

Clinical Practice

Rheumatology.

\footnotetext{
Competing interests

The author declared no

competing interests.

www.nature.com/clinicalpractice doi:10.1038/ncprheum0992
}

possible that deleting or suppressing the activity of either B cells or T cells alone is insufficient to control SLE in a heterogeneous population of subjects. Alternatively, as the spectrum of activities of these agents in humans is not completely delineated, it is possible that a pathogenic population of lymphocytes is not targeted by these agents. In this regard, it is known that rituximab does not delete all human $B$ lineage cells; that BAFF (the target of belimumab) is not essential for the survival of all human B-cell subsets; and that costimulation blockade with abatacept might not interfere with activation of all T-cell subsets. In addition, as the 'immunopharmacodynamics' of these agents have not been established specifically in patients with SLE, no effective dosage regimens have been determined. The most appropriate trial design, patient selection, biomarkers and outcome measures to employ to detect and document clinical benefit in SLE have also not been determined; without this information, development of new treatments for SLE will be challenging.

The apparent difficulty of developing therapeutics for SLE is often compared to the rather easier development of biologics for use in rheumatoid arthritis (RA). It is important to emphasize that the development of biologics in RA was only easy in retrospect; many trials failed, and many agents were-and continue to be-rejected in the quest for safer and cheaper new agents for RA. The pathway to new agents for SLE is likely to be similar, with a number of fits and starts until a successful agent defines the nature of the approach that will succeed. For the sake of people with SLE, who need better therapies, it is hoped that neither pharmaceutical companies nor clinical investigators will become discouraged before the best pathway to safe and effective new therapeutics for SLE is discovered. 